\title{
Transcranial doppler ultrasound to assess cerebrovascular reactivity: reliability, reproducibility and effect of posture.
}

Transcranial Doppler ultrasound (TCD) allows measurement of blood flow velocities in the intracranial vessels, and can be used to assess cerebral vasodilator responses to a hypercapnic stimulus. The reliability of this technique has not been established, nor is there agreement about whether the technique should be performed in sitting or lying. We tested the intra- and inter-rater reliability of measures of cerebrovascular reactivity (CVR) in 10 healthy adults, in sitting and lying postures. Participants underwent triplicate bilateral ultrasound assessment of flow velocities in the middle cerebral arteries whilst sitting and lying supine prior to and during inhalation of Carbogen ( $5 \% \mathrm{CO}_{2}$, $95 \% \mathrm{O}_{2}$ ) for $2 \mathrm{~min}$. This procedure was performed twice by each of two raters for a total of four sessions. CVR was calculated as the difference between baseline and the peak blood flow velocity attained during $\mathrm{CO}_{2}$ inhalation. Intraclass correlation coefficients (ICCs) for intra-rater reliability were greater sitting than lying for both raters (e.g. Rater 1 ICC sitting $=0.822$, lying $=0.734$ ), and inter-rater reliability was also greater in sitting (e.g. sitting ICC $=0.504$, lying $=0.081$ ). These results suggest that assessment of CVR using TCD should be performed with participants sitting in order to maximise CVR measurement reliability. 
Transcranial Doppler ultrasound to assess cerebrovascular reactivity: reliability, reproducibility and effect of posture.

\author{
${ }^{1}$ Michelle N McDonnell $\bowtie$ \\ ${ }^{2}$ Narelle M Berry \\ ${ }^{2}$ Mark A Cutting \\ ${ }^{3}$ Hannah A Keage \\ ${ }^{2}$ Jonathan D Buckley \\ ${ }^{2}$ Peter RC Howe
}

${ }^{1}$ School of Health Sciences, University of South Australia, Adelaide, Australia

${ }^{2}$ Nutritional Physiology Research Centre, University of South Australia

${ }^{3}$ School of Psychology' Social Work and Social Policy, University of South Australia

Abbreviated title: TCD to assess cerebrovascular reactivity

$\bowtie$ Dr Michelle N McDonnell

School of Health Sciences

University of South Australia

GPO Box 2471

Adelaide SA 5001

Australia

Email: michelle.mcdonnell@unisa.edu.au

Phone: +61 883021684

Fax: +61 883022168 


\section{Abstract}

Transcranial Doppler ultrasound (TCD) allows measurement of blood flow velocities in the intracranial vessels, and can be used to assess cerebral vasodilator responses to a hypercapnic stimulus. The reliability of this technique has not been established, nor is there agreement about whether the technique should be performed in sitting or lying. We tested the intra- and inter-rater reliability of measures of cerebrovascular reactivity (CVR) in 10 healthy adults, in sitting and lying postures. Participants underwent triplicate bilateral ultrasound assessment of flow velocities in the middle cerebral arteries whilst sitting and lying supine prior to and during inhalation of Carbogen $\left(5 \% \mathrm{CO}_{2}, 95 \% \mathrm{O}_{2}\right)$ for $2 \mathrm{~min}$. This procedure was performed twice by each of two raters for a total of four sessions. CVR was calculated as the difference between baseline and the peak blood flow velocity attained during $\mathrm{CO}_{2}$ inhalation. Intraclass correlation coefficients (ICCs) for intra-rater reliability were greater sitting than lying for both raters (e.g. Rater $1 \mathrm{ICC}$ sitting $=0.822$, lying $=$ 0.734), and inter-rater reliability was also greater in sitting (e.g. sitting ICC $=0.504$, lying $=0.081$ ). These results suggest that assessment of CVR using TCD should be performed with participants sitting in order to maximise CVR measurement reliability. 


\section{Introduction}

2 Transcranial Doppler (TCD) sonography allows real time measurement of blood flow velocity in

3 intracranial arteries with high temporal resolution and has been used for over two decades to assess

4 cerebral haemodynamics in healthy adults and in disease states. TCD can also be utilised to assess

5 cerebrovascular reactivity (CVR), i.e. the vasodilation of cerebral arterioles in response to a

6 physiological stimulus such as an increase in the partial pressure of arterial $\mathrm{CO}_{2}$ (hypercapnia)

7 (Ainslie and Duffin, 2009, Willie et al., 2011). Several reports suggest that the cerebral vasodilator

8 response to hypercapnia depends on the integrity of the vascular endothelium (Silvestrini et al.,

92000 , Krainik et al., 2005, Lavi et al., 2003) such that $\mathrm{CO}_{2}$ induced CVR could serve as a surrogate

10 marker of endothelial function in cerebral arteries (Lavi et al., 2006). However, the reliability of the

11 CVR method, particularly under differing postural conditions (i.e. lying vs sitting) has not been fully

12 investigated.

Differing methods can be used to induce a cerebral arteriolar vasodilatory response, such as hypercapnia (using steady state breathing during 5\% CO2 exposure or breath holding techniques) or medications such as acetazolamide (Dahl et al., 1995). Regardless of the stimulus, CVR is taken as the resultant change in mean blood flow velocity from a pre-stimulus baseline (Kernan et al., 2000, Willie et al., 2011). Mean blood flow velocity is typically measured in a conduit vessel such as the middle cerebral artery (MCA) which supplies a large proportion of brain tissue but will itself dilate minimally in response to the stimulus (Serrador et al., 2000). Thus changes of blood flow velocity in the MCA reflect changes in resistance to flow in the distal vasculature, i.e. arterioles in brain regions supplied by the MCA.

Repeatability of TCD measurements of MCA blood flow velocities was established nearly two decades ago. Demolis and colleagues confirmed that both intra-rater reproducibility and inter-rater reliability were satisfactory with strong correlations ( $r \geq 0.9$ in both cases)(Demolis et al., 1993). This 
study also established that mean velocities in the MCA vary with time of day, with significantly lower velocities recorded at 11 am compared with $8 \mathrm{am}$, and these variations in flow velocity follow a similar time course to diurnal variations in mean arterial pressure. This highlights the importance of conducting repeat experiments at the same time of day.

Reliability of CVR measures has previously been reported with the aim of comparing different methods of inducing hypercapnia. Intra-rater reliability of CVR measures was superior when induced by inhalation of $5 \% \mathrm{CO}_{2}$ but this study did not address the reliability of different raters or different postures (Totaro et al., 1999). Others have compared measurements of CVR in lying and sitting postures and found that baseline and peak cerebral blood flow velocity (CBV) were lower in sitting compared to lying, but reproducibility of the method in sitting was not investigated (Mayberg et al., 1996).

\section{This study aimed to determine the intra- and inter-rater reliability of measures of CVR in} bilateral MCAs of healthy adult participants in sitting versus lying positions. Investigation of posture was performed due to the availability of ultrasound probes attached to a headband, allowing this technique to be performed sitting and lying supine. Sitting may be preferable for some participants, particularly the elderly who may find lying supine whilst holding a breathing tube and mouthpiece uncomfortable.

\section{Methods}

\section{Participants}

Ten adult participants (aged 20-64, mean age 33.8 \pm 13.4 years [mean \pm SD]) were recruited from staff and students of the host institution after giving their written informed consent. The study was approved by the institutional Human Research Ethics Committee. Participants were excluded if they had cardiovascular or renal disease, a history of diabetes, stroke and/or neurological 
conditions, were smokers or on nicotine replacement therapy, or were taking anticholinergic or psychotropic medications. Participants refrained from eating two hours prior to testing and caffeine four hours prior to testing.

\section{Apparatus}

Bilateral MCA blood flow velocities were measured with a Multigon TOC2M Neurovision TCD system (Multigon Industries Inc, New York, USA) using two 2-MHz transducer probes securely mounted on an adjustable headband. Time-averaged mean blood flow velocity measures $(\mathrm{cm} / \mathrm{s})$ were obtained every second (i.e. $1 \mathrm{~Hz}$ sampling rate) and stored for off-line analysis.

\section{Procedure}

Participants attended an air-conditioned laboratory on four separate occasions at the same time of day, with each visit separated by one week. Assessments in both the lying and sitting positions were conducted on two occasions by Rater $1(\mathrm{MMcD})$ and on two occasions by Rater 2 (MAC); both raters had recently performed at least 25 assessments. The order of assessments, either sitting or lying supine, was randomised between raters and sessions. On arrival, the headband that holds the two 2.0 MHz transducers was placed over the head and adjusted for a comfortably firm fit. The 2$\mathrm{MHz}$ transducers were then centred on the temple, adjusted until maximal amplitude signals were obtained bilaterally, at which time they were secured in the headband to ensure stability of the signals for the duration of the experiment. The precise positioning and recording of mean flow velocity in each MCA was performed as previously described (Aaslid et al., 1982) through the transtemporal windows, above the zygomatic arch. Participants breathed room air through a 2-way nonrebreathing valve and mouth piece with a nose-clip attached and were either seated comfortably in a chair with arm-rests, feet flat on the floor, or lying supine with one pillow for at least 10 minutes prior to commencing the testing. Participants were advised to perform "relaxed breathing, in and out through the mouth". 
After at least 10 minutes of breathing room air through the mouthpiece, measurement of CVR was performed during hypercapnia induced by breathing a mixture of $5 \% \mathrm{CO}_{2}-95 \% \mathrm{O}_{2}$ (Carbogen) from a spirometer. This method was used in preference to breath holding or controlled hypoventilation to avoid concomitant hypoxia which can influence the ventilatory response (Ainslie and Duffin, 2009) and it has been shown to be more reproducible than other methods when performed by the one rater (Totaro et al., 1999). Beat-to-beat changes in mean CBV were measured bilaterally during a 30-second baseline period, then breathing the Carbogen mixture for 2 minutes. Each test was then followed by a 2 minute rest period breathing room air again. This procedure was repeated three times both sitting and lying (counterbalanced) separated by a 10 minute rest period after changing positions. Participants returned the following week at the same time of day to repeat the study with the same rater, then on two subsequent occasions with the other rater

83 (counterbalanced).

\section{$84 \quad$ Data analysis and statistics}

The sample size of 10 participants was chosen in accordance with other reliability studies involving TCD (Harrer et al., 2011, Brodie et al., 2009). Recordings of bilateral CBV waveforms were averaged over the 30s baseline period in each of the triplicate assessments in each position (for each of the four separate testing sessions). The CBV waveforms obtained during the 2 min periods of hypercapnia were plotted against time and a spline curve was fitted ( $20 \%$ smoothing, LOESS filter) to aid determination of the peak blood flow velocity (Table Curve 2D, Systat Software Inc, CA, USA)(Figure 1). Calculation of CVR involves comparing beat to beat changes in CBV to a pre-stimulus baseline (Willie et al., 2011, Wijnhoud et al., 2006) and is calculated:

$\% C V R=100 \times($ peak CBV -baseline CBV)/baseline CBV. and the average of the three values was used for further analysis. 
All data were analysed using SPSS software (PASW Statistics 18.0) and graphs were created in

97

98

99

100

101

102

103

104

Sigmaplot 12 (Systat Software, Inc). Paired t-tests were used to verify differences between left and right sides and between sitting and lying CVR measures. Inter- and intra-rater reliability was established using intra-class correlation coefficients (ICC). As only two raters were used to score an average rating of CVR which is analysed in a two-way ANOVA model, this is evaluated using a Case 3, average rating or $(3,1)$ ICC model, In accordance with Shrout and Fleiss (1979). Results were considered significant at $P<0.05$.

\section{Results}

10 participants were enrolled in the study with 8 participants completing all four sessions. Two participants (authors $\mathrm{MMcD}$ and $\mathrm{MAC}$ ) attended only two sessions, both with the same rater; thus intra-rater reliability data was obtained from 9 participants by each rater and inter-rater reliability was assessed for 8 participants.

Data were collected bilaterally at each session and values for left and right MCAs were compared. Depth of insonation was similar for left and right sides and between postures (e.g. $50.3 \pm$ $4.1 \mathrm{~mm}$ in lying, $50.2 \pm 4.1 \mathrm{~mm}$ in sitting). There were no differences between hemispheres when data from both testing sessions for both raters were combined (sitting $P=0.27$, lying $P=0.46$ ), allowing this data to be combined for further analysis (i.e. left and right MCA velocities were collapsed for all subsequent analyses).

\section{Differences between lying and sitting}

Individual CVR data from each test performed by both raters at both sessions were collapsed relative to sitting or lying. Differences in variability between the two positions were explored using Coefficients of Variation (CV) to quantify whether there was greater variation if measures were taken sitting rather than lying supine. CVs were calculated separately for sitting and lying measures 
obtained from each participant on each test occasion. There were no differences in variability of CVR measures obtained sitting versus lying (sitting average $C V=35.2 \pm 6.6 \%$; lying average $C V=36.7 \pm$ 8.1\%; paired t-test $P=0.5$ ).

\section{Intra-rater reliability}

Measures obtained sitting and lying at two testing sessions by Rater 1 and Rater 2 were analysed using intraclass correlation coefficients to compare CVR measurements between sessions. Results for Rater 1 (Figure 2) illustrate superior reproducibility between sessions when measured sitting (ICC $(3,1)=0.822 ; P<0.001)$ compared with lying $(\operatorname{ICC}(3,1)=0.734 ; P<0.05)$.

Measures for Rater 2 were comparable, as shown in Figure 3, with greater reproducibility in sitting $(\operatorname{ICC}(3,1)=0.463 ; P=0.12)$ compared with lying, although the correlations were nonsignificant $(\operatorname{ICC}(3,1)=0.015 ; \mathrm{P}=0.49)$.

\section{Inter-rater reliability}

To demonstrate inter-rater reliability for both sitting and lying postures, data for each side (left and right) and each session (Session 1 and 2) were combined so that CVR values obtained by Rater 1 and Rater 2 could be compared as in Figure 4. Intraclass correlation coefficients were determined and were significant for sitting $(\operatorname{ICC}(3,1)=0.504 ; P=0.028)$ but not lying $(\operatorname{ICC}(3,1)=0.081 ; P=0.41)$.

\section{Discussion}

Assessment of CVR in healthy adults can be achieved with bilateral recording of CBV in the MCA with acceptable reproducibility and inter-rater reliability. This is the first study to demonstrate that measures of CVR obtained sitting were more reproducible than whilst lying. The reproducibility measures obtained were superior to those obtained by Totaro and colleagues (Totaro et al., 1999) (ICC $=0.43$ when measures were repeated by a single investigator after 24 hours). Reproducibility 
measures for both raters were greater in sitting compared with lying $(\operatorname{ICC}(3,1)=0.822$ and 0.463 in sitting respectively for both raters ) which shows a strong agreement between sessions. Intra-rater reliability was greater for the more recently experienced sonographer, Rater 1 , confirming previous reports (Martin et al., 1993, Shen et al., 1999). Inter-rater reliability was only fair, indicating that the same rater should be used where possible for measurements in research trials and clinical settings. It is important to note that these values for reliability and reproducibility are valid for measurement of hypercapnia at $5 \% \mathrm{CO}_{2}$ and $95 \% \mathrm{O}_{2}$, providing a strong vasodilatory stimulus but we acknowledge that for near maximal vasodilation to occur, $10 \% \mathrm{CO}_{2}$ may be required (Goode et al., 2009).

We have recently shown (Wong et al., 2012) that regular dietary supplementation with a wild green oat extract can enhance systemic endothelial function assessed by the technique flow mediated dilatation of the brachial artery and cerebral endothelial function assessed in the MCA by CVR to hypercapnia. Even though these responses were of similar magnitude ( $\sim 40 \%$ increase in both cases) they were not correlated within individuals, indicating the likely independence of underlying mechanisms and pathophysiological changes in endothelial function between systemic and cerebral arteries. Thus, while the technique of flow mediated dilatation of the brachial artery is well established, TCD has the potential to determine the efficacy of therapeutic treatments to improve vascular health. The present study provides evidence of good intra-rater reliability, particularly with an experienced sonographer, but only acceptable inter-rater reliability using Carbogen as the hypercapnic stimulus to measure changes in CVR within-individuals in response to therapeutic interventions. The results of this study suggest that performing the test in sitting is preferable to lying to assess changes in cerebral endothelial vasodilator function which may be appropriate for elderly or disabled populations who may be involved in research investigating dietary or behavioural interventions to improve cerebrovascular function. 
The technique of TCD to assess changes in cerebral blood flow velocity, although widely used, does have a known limitation, in that we cannot prove that the diameter of the MCA does not change during the period of hypercapnia. The method used here and by others assumes that the MCA diameter was constant during hypercapnia (Serrador et al., 2000). A number of studies have demonstrated that the diameter of the MCA does not change significantly during craniotomy (Giller et al., 1993), after the release of thigh cuffs to assess cerebral autoregulation (Newell et al., 1994) or a similar TCD technique using the Doppler power signal to measure MCA diameter (Poulin et al., 1996). However, there have been reports in animal and human studies that small changes in vessel diameter are possible, particularly during hypocapnia (Du Boulay and Symon, 1971, Du Boulay et al., 1972).

There are several limitations to our study. We only used two raters and additional raters may provide more accurate measures of ICCs. Further, we did not measure blood pressure of these healthy individuals, nor did we relate our measurement of $\mathrm{CVR}$ to end-tidal $\mathrm{CO}_{2}$ by performing gas analysis on expired air during the experiments. Our results, therefore, are limited to relative changes in $\mathrm{CBV}$ in the $\mathrm{MCA}$ in response to $5 \% \mathrm{CO}$, rather than relative increase in $\mathrm{CBV}$ per kiloPascal rise in end-tidal $\mathrm{CO}_{2}$ (Wijnhoud et al., 2006). The important metric to record when assessing CVR is the beat-to-beat change in CBV from a pre-stimulus baseline (Willie et al., 2011) and the method we have used for this and other clinical studies satisfies this requirement and avoids the subject discomfort which can occur with administration of $\geq 8 \% \mathrm{CO}_{2}$ (Ainslie and Duffin, 2009). This method has been used by others to assess cerebrovascular endothelial function (Wijnhoud et al., 2006, Schwertfeger et al., 2006) and has been shown to be preferable to normalizing to absolute increase in end-tidal $\mathrm{CO}_{2}$ in predicting stroke and TIA risk in patients with carotid artery stenosis and occlusion (Markus and Cullinane, 2001).

The data from this study indicates that the assessment of CVR using TCD provides more reliable values when performed sitting compared with lying, particularly with a more experienced 

lying to maximise CVR responses and to optimise inter- and intra-rater reliability. 
195

196

197

198

199

200

201

202

203

204

205

206

207

208

209

210

211

212

213

214

215

216

217

218

219

220

221

222

223

224

225

226

227

\section{References}

AASLID, R., MARKWALDER, T. M. \& NORNES, H. 1982. Noninvasive transcranial Doppler ultrasound recording of flow velocity in basal cerebral arteries. J Neurosurg, 57, 769-74.

AINSLIE, P. N. \& DUFFIN, J. 2009. Integration of cerebrovascular CO2 reactivity and chemoreflex control of breathing: mechanisms of regulation, measurement, and interpretation. Am J Physiol Regul Integr Comp Physiol, 296, R1473-95.

BRODIE, F. G., ATKINS, E. R., ROBINSON, T. G. \& PANERAI, R. B. 2009. Reliability of dynamic cerebral autoregulation measurement using spontaneous fluctuations in blood pressure. Clin Sci (Lond), 116, 513-20.

DAHL, A., RUSSELL, D., ROOTWELT, K., NYBERG-HANSEN, R. \& KERTY, E. 1995. Cerebral vasoreactivity assessed with transcranial Doppler and regional cerebral blood flow measurements. Dose, serum concentration, and time course of the response to acetazolamide. Stroke, 26, 2302-6.

DEMOLIS, P., CHALON, S. \& GIUDICELLI, J. F. 1993. Repeatability of transcranial Doppler measurements of arterial blood flow velocities in healthy subjects. Clin Sci (Lond), 84, 599604.

DU BOULAY, G., SYMON, L., SHAH, S., DORSCH, N. \& ACKERMAN, R. 1972. Cerebral arterial reactivity and spasm after subarachnoid haemorrhage. Proc $R$ Soc Med, 65, 80-2.

DU BOULAY, G. H. \& SYMON, L. 1971. The anaesthetist's effect upon the cerebral arteries. Proc $R$ Soc Med, 64, 77-80.

GILLER, C. A., BOWMAN, G., DYER, H., MOOTZ, L. \& KRIPPNER, W. 1993. Cerebral arterial diameters during changes in blood pressure and carbon dioxide during craniotomy. Neurosurgery, 32, 737-41; discussion 741-2.

GOODE, S. D., KRISHAN, S., ALEXAKIS, C., MAHAJAN, R. \& AUER, D. P. 2009. Precision of cerebrovascular reactivity assessment with use of different quantification methods for hypercapnia functional MR imaging. AJNR Am J Neuroradiol, 30, 972-7.

HARRER, J. U., VALAIKIENE, J., KOCH, H., KNORR, R., HORN, M., ICKENSTEIN, G., BOGDAHN, U. \& SCHLACHETZKI, F. 2011. Transcranial perfusion sonography using a low mechanical index and pulse inversion harmonic imaging: reliability, inter-/intraobserver variability. Ultraschall Med, 32 Suppl 1, S95-101.

KERNAN, W. N., VISCOLI, C. M., BRASS, L. M., MAKUCH, R. W., SARREL, P. M., ROBERTS, R. S., GENT, M., ROTHWELL, P., SACCO, R. L., LIU, R. C., BODEN-ALBALA, B. \& HORWITZ, R. I. 2000. The stroke prognosis instrument II (SPI-II) : A clinical prediction instrument for patients with transient ischemia and nondisabling ischemic stroke. Stroke, 31, 456-62.

KRAINIK, A., HUND-GEORGIADIS, M., ZYSSET, S. \& VON CRAMON, D. Y. 2005. Regional impairment of cerebrovascular reactivity and BOLD signal in adults after stroke. Stroke, 36, 1146-52. 
LAVI, S., EGBARYA, R., LAVI, R. \& JACOB, G. 2003. Role of nitric oxide in the regulation of cerebral blood flow in humans: chemoregulation versus mechanoregulation. Circulation, 107, 1901-5.

LAVI, S., GAITINI, D., MILLOUL, V. \& JACOB, G. 2006. Impaired cerebral CO2 vasoreactivity: association with endothelial dysfunction. Am J Physiol Heart Circ Physiol, 291, H1856-61.

MARKUS, H. \& CULLINANE, M. 2001. Severely impaired cerebrovascular reactivity predicts stroke and TIA risk in patients with carotid artery stenosis and occlusion. Brain, 124, 457-67.

MARTIN, N. A., THOMAS, K. M. \& CARON, M. 1993. Transcranial Doppler--techniques, application, and instrumentation. Neurosurgery, 33, 761-4.

MAYBERG, T. S., LAM, A. M., MATTA, B. F. \& VISCO, E. 1996. The variability of cerebrovascular reactivity with posture and time. J Neurosurg Anesthesiol, 8, 268-72.

NEWELL, D. W., AASLID, R., LAM, A., MAYBERG, T. S. \& WINN, H. R. 1994. Comparison of flow and velocity during dynamic autoregulation testing in humans. Stroke, 25, 793-7.

POULIN, M. J., LIANG, P. J. \& ROBBINS, P. A. 1996. Dynamics of the cerebral blood flow response to step changes in end-tidal PCO2 and PO2 in humans. J Appl Physiol, 81, 1084-95.

SCHWERTFEGER, N., NEU, P., SCHLATTMANN, P., LEMKE, H., HEUSER, I. \& BAJBOUJ, M. 2006. Cerebrovascular reactivity over time course in healthy subjects. J Neurol Sci, 249, 135-9.

SERRADOR, J. M., PICOT, P. A., RUTT, B. K., SHOEMAKER, J. K. \& BONDAR, R. L. 2000. MRI measures of middle cerebral artery diameter in conscious humans during simulated orthostasis. Stroke, 31, 1672-8.

SHEN, Q., STUART, J., VENKATESH, B., WALLACE, J. \& LIPMAN, J. 1999. Inter observer variability of the transcranial Doppler ultrasound technique: impact of lack of practice on the accuracy of measurement. J Clin Monit Comput, 15, 179-84.

SHROUT, P. E. \& FLEISS, J. L. 1979. Intraclass correlations: uses in assessing rater reliability. Psychol Bull, 86, 420-8.

SILVESTRINI, M., VERNIERI, F., PASQUALETTI, P., MATTEIS, M., PASSARELLI, F., TROISI, E. \& CALTAGIRONE, C. 2000. Impaired cerebral vasoreactivity and risk of stroke in patients with asymptomatic carotid artery stenosis. JAMA, 283, 2122-7.

TOTARO, R., MARINI, C., BALDASSARRE, M. \& CAROLEI, A. 1999. Cerebrovascular reactivity evaluated by transcranial Doppler: reproducibility of different methods. Cerebrovasc Dis, 9, 142-5.

WIJNHOUD, A. D., KOUDSTAAL, P. J. \& DIPPEL, D. W. 2006. Relationships of transcranial blood flow Doppler parameters with major vascular risk factors: TCD study in patients with a recent TIA or nondisabling ischemic stroke. J Clin Ultrasound, 34, 70-6.

WILLIE, C. K., COLINO, F. L., BAILEY, D. M., TZENG, Y. C., BINSTED, G., JONES, L. W., HAYKOWSKY, M. J., BELLAPART, J., OGOH, S., SMITH, K. J., SMIRL, J. D., DAY, T. A., LUCAS, S. J., ELLER, L. K. \& 
AINSLIE, P. N. 2011. Utility of transcranial Doppler ultrasound for the integrative assessment of cerebrovascular function. J Neurosci Methods, 196, 221-37.

WONG, R. H. X., BERRY, N. M., BUCKLEY, J. D., COATES, A. M. \& HOWE, P. R. C. 2012. Regular consumption of a wild green oat extract enhances systemic and cerebral vasodilator function. Hypertension, (in press). 
Figure 1 Beat-to-beat cerebral blood flow velocities (CBV) obtained during the 2-min period of

274 hypercapnia were plotted and fitted with a spline curve, enabling the peak of the curve to be identified as peak CBV

Figure 2 Correlations between \%CVR measures taken on two occasions by Rater 1 in sitting (left, $277 \operatorname{ICC}(3,1)=0.822)$ and lying (right, ICC $(3,1)=0.734)$. Simple linear regression lines are shown, using the least squares method (Sigmaplot 12).

Figure 3 Correlations between \%CVR measures taken on two occasions by Rater 2 in sitting (left, $\operatorname{ICC}(3,1)=0.463)$ and lying (right, ICC $(3,1)=0.015)$. Simple linear regression lines are shown, using the least squares method (Sigmaplot 12).

Figure 4 Correlations between \%CVR measures obtained across both sessions by Rater 1 compared with Rater 2 for sitting (left, ICC $(3,1)=0.504$ ) and lying (right, ICC $(3,1)=0.081$ ). Simple linear regression lines are shown, using the least squares method (Sigmaplot 12 ). 


\section{Figure 1}

Application of curve fitting to CBFV data

Beat-to-beat cerebral blood flow velocities (CBV) obtained during the 2-min period of hypercapnia were plotted and fitted with a spline curve, enabling the peak of the curve to be identified as peak CBV

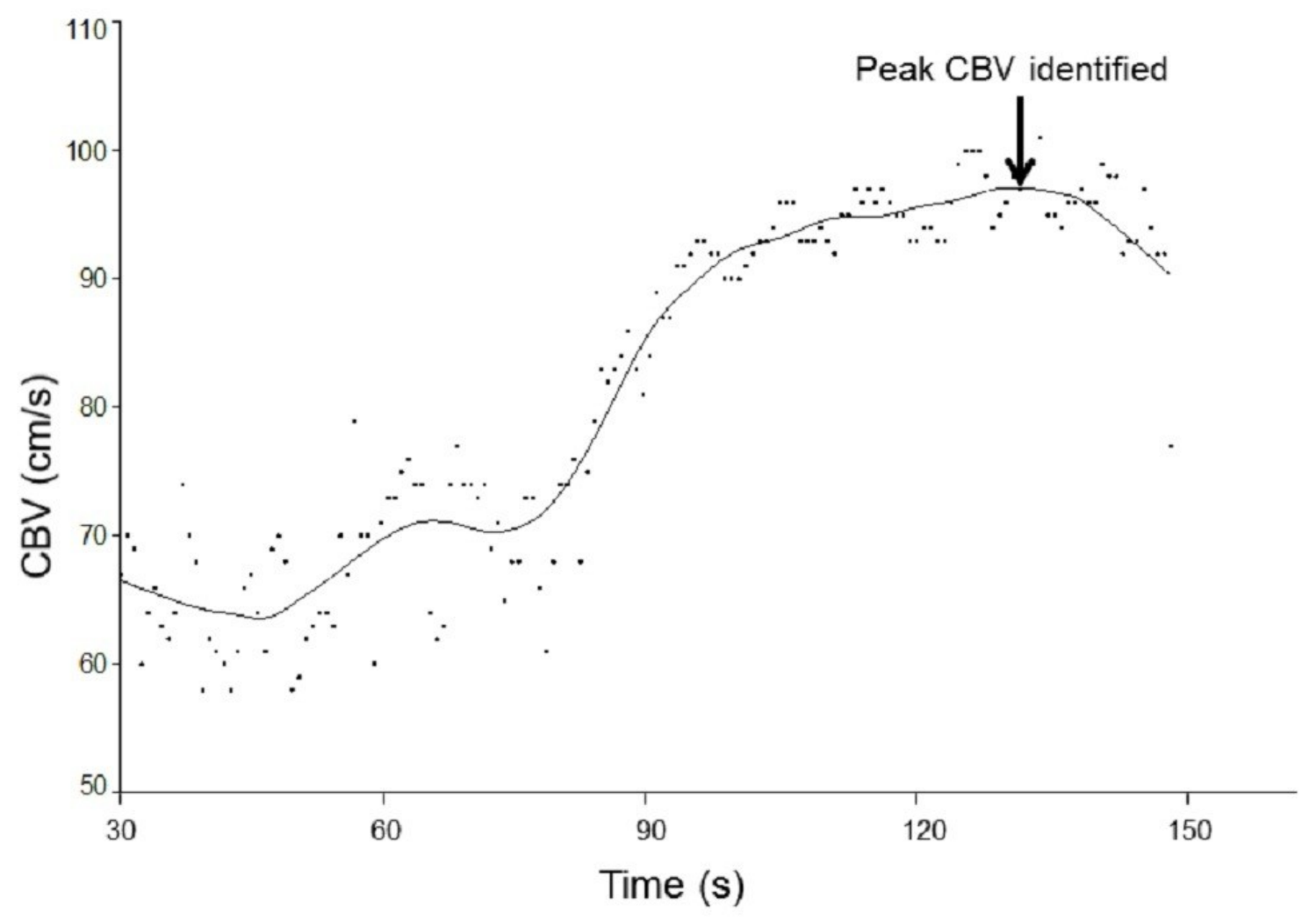




\section{Figure 2}

ICCs rater 1 sitting and lying

Correlations between \%CVR measures taken on two occasions by Rater 1 in sitting (left, ICC $(3,1)=$ 0.822) and lying (right, ICC $(3,1)=0.734)$. Simple linear regression lines are shown, using the least squares method (Sigmaplot 12).
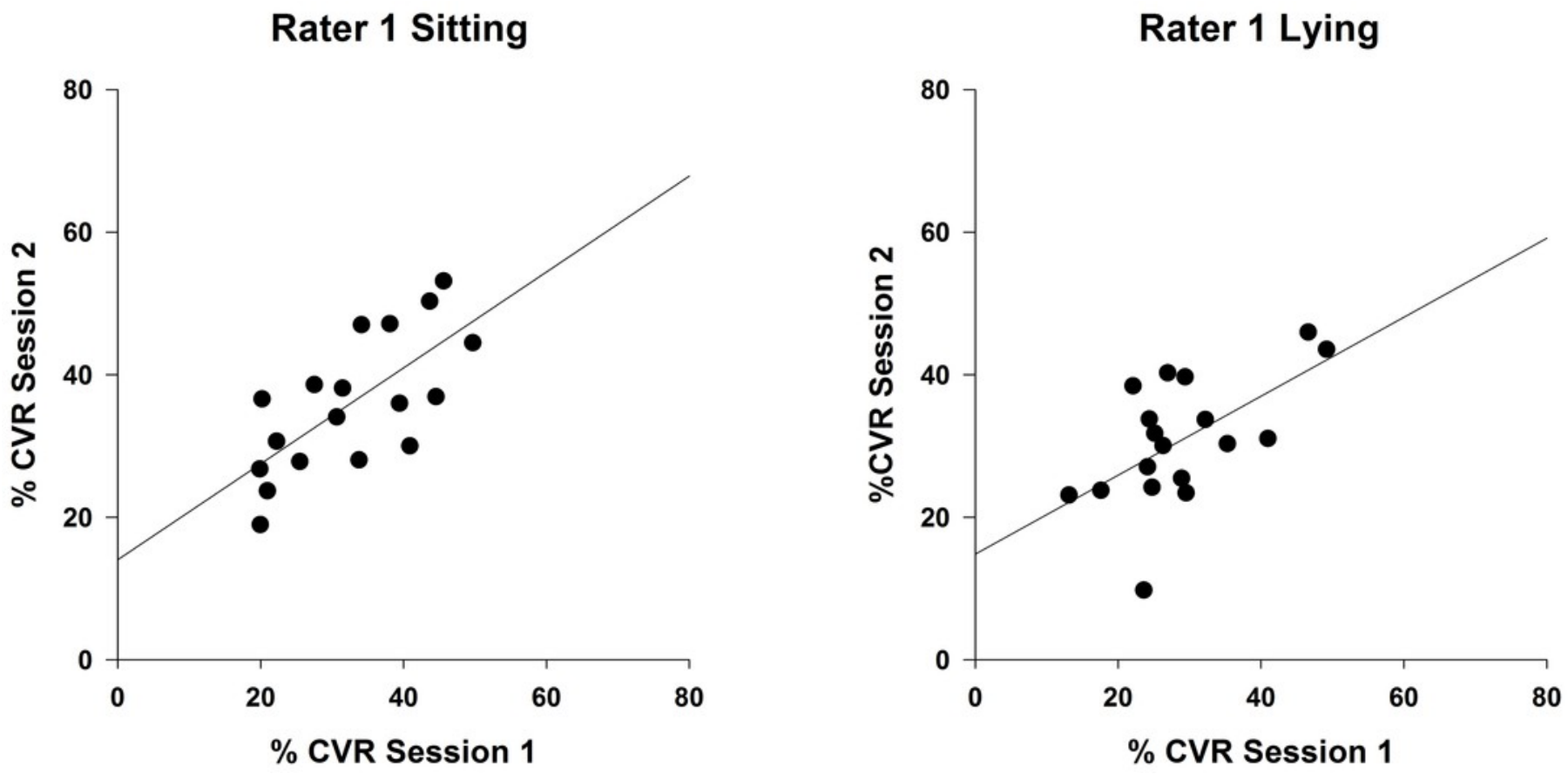


\section{Figure 3}

Intra-rater reliability correlations in sitting and lying, Rater 2

Correlations between \%CVR measures taken on two occasions by Rater 2 in sitting (left, ICC $(3,1)=$ 0.463 ) and lying (right, ICC $(3,1)=0.015)$. Simple linear regression lines are shown, using the least squares method (Sigmaplot 12).
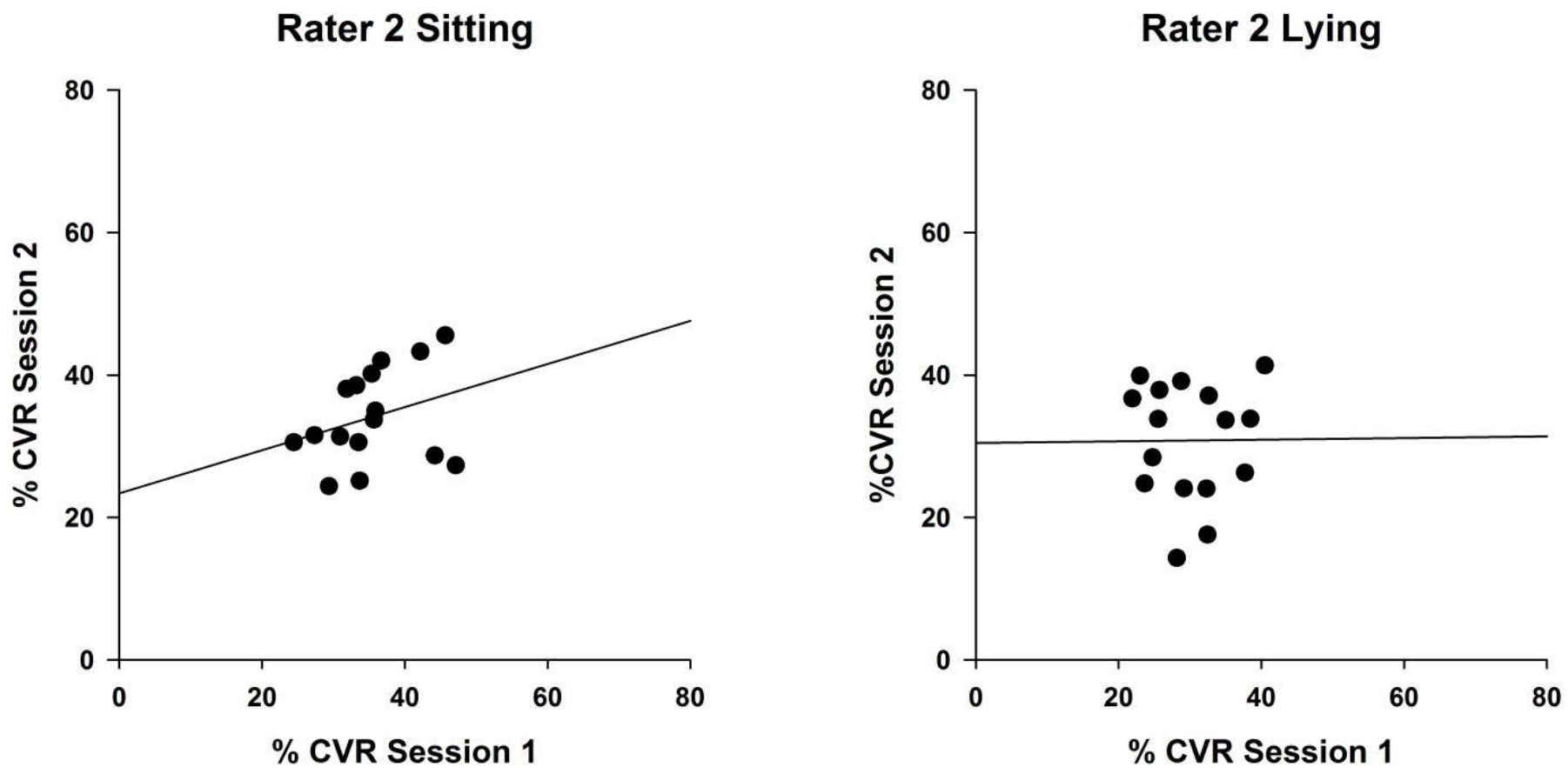


\section{Figure 4}

Inter-rater reliability comparisons showing superior reliability in sitting

Correlations between \%CVR measures obtained across both sessions by Rater 1 compared with Rater 2 for sitting (left, ICC $(3,1)=0.504)$ and lying (right, ICC $(3,1)=0.081)$. Simple linear regression lines are shown, using the least squares method (Sigmaplot 12).

Sitting

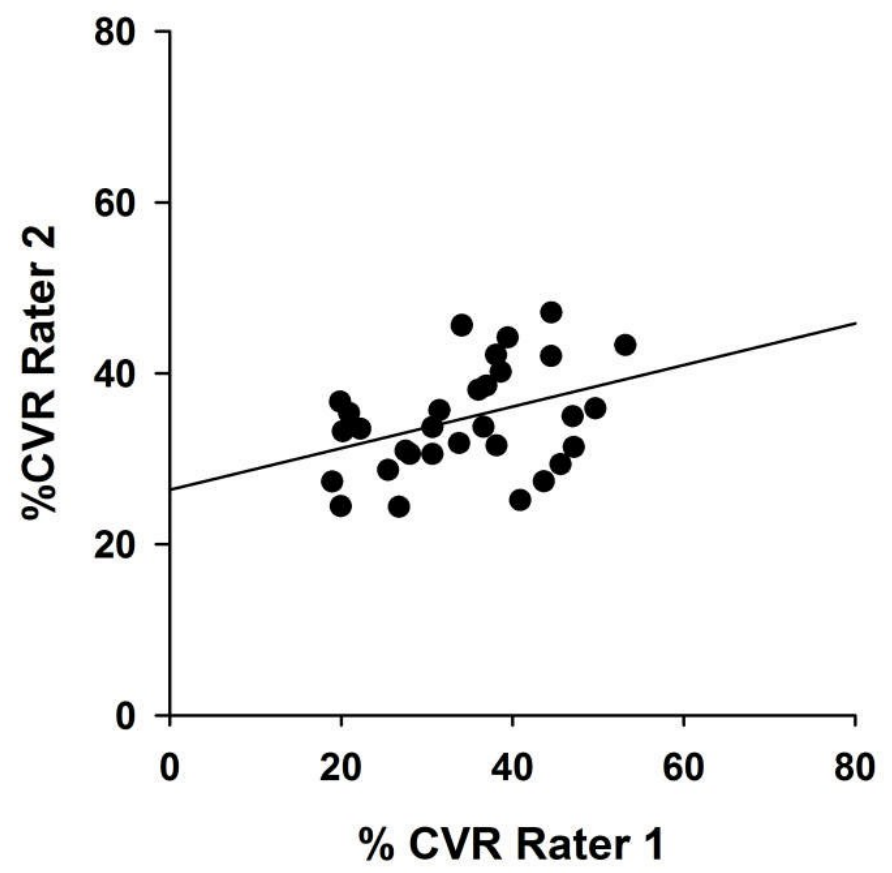

Lying

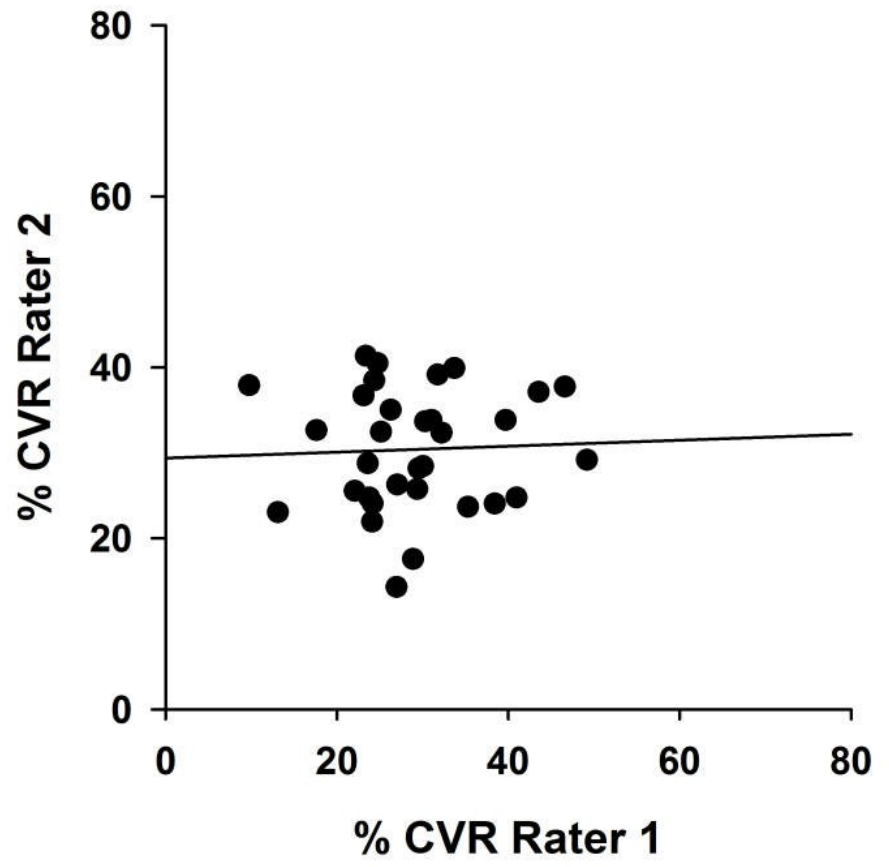

